\title{
Analytical one-dimensional model for laser-induced ultrasound in planar optically absorbing layer
}

\author{
Erika Svanström*, Tomas Linder, Torbjörn Löfqvist
}

EISLAB, Department of Computer Science and Electrical Engineering

Luleå University of Technology, SE-971 87 Luleå, Sweden

\begin{abstract}
Ultrasound generated by means of laser-based photoacoustic principles are in common use today and applications can be found both in biomedical diagnostics, non-destructive testing and materials characterisation. For certain measurement applications it could be beneficial to shape the generated ultrasound regarding spectral properties and temporal profile. To address this, we studied the generation and propagation of laser-induced ultrasound in a planar, layered structure. We derived an analytical expression for the induced pressure wave, including different physical and optical properties of each layer. A Laplace transform approach was employed in analytically solving the resulting set of photoacoustic wave equations. The results correspond to simulations and were compared to experimental results. To enable the comparison between recorded voltage from the experiments and the calculated pressure we employed a system identification procedure based on physical properties of the ultrasonic transducer to convert the calculated acoustic pressure to voltages. We found reasonable agreement between experimentally obtained voltages and the voltages determined from the calculated acoustic pressure, for the samples studied. The system identification procedure was found to be unstable, however, possibly from violations of material isotropy assumptions by film adhesives and coatings in the experiment. The presented analytical model can serve as a basis when addressing the inverse problem of shaping an acoustic pulse from absorption of a laser pulse in a planar layered structure of elastic materials.
\end{abstract}

Keywords: photoacustic, laser-ultrasound, thermoelastic, optical absorption, thin film, polymer

\section{Introduction}

The use of laser based photoacoustic principles in generation of ultrasound is an efficient way to generate ultrasound pulses of high frequencies and large bandwidths. These properties has been utilised foremost in biomedical imaging and

\footnotetext{
* Corresponding author

Email address: erika.svanstrom@ltu.se (Erika Svanström)
} 
diagnosis [1] but also in e.g. materials characterisation [2], or non-destructive testing [3] or other engineering applications [4]. The technique rests on absorption of an incident laser light pulse and energy conversion through a thermoelastic process resulting in ultrasonic waves [5]. An objective in earlier studies has been to create a high conversion efficiency situation, i.e. maximizing the ultrasound pulse energy by optimizing the light absorption and energy conversion in the light absorbing layer. This is commonly achieved by opaque light absorbing films or structures as e.g. metal thin films [6], light absorbing polymer thin films $[7,8]$, optically absorbing gold nano structures $[9,10]$, or thin layers of carbon nanotube composites [11].

However, in some applications a desirable feature would be to use a semitransparent layer that partly absorbs and partly transmits the incoming laser radiation. This could be useful in applications where a traditional, pulse-echo technique is used in conjunction with e.g. photoacoustic tomography. In this case the optical absorption is only partial and the possibility appears to construct the absorbent such that the resulting ultrasound is influenced by the physical layout and material properties of the absorbent. Planar, layered absorbents has been studied earlier within the field of photoacostic spectroscopy. Sun and Diebold [12] modelled generation of ultrasonic waves from a photoacoustic source consisting of planar, alternating layers of weakly light-absorbing solid and transparent fluid layers. An amplitude-modulated laser generated acoustic waves at specific resonance frequencies by means of acoustic interference in the layers. In order to shape the resulting ultrasonic pulse, light absorbing or transparent layers may be interleaved with layers having different optical absorption coefficients, thickness's and elastic properties. The stack will then operate basically as a acoustic filter. Hu et al. [13] modelled layers of arbitrary physical properties and calculated the photoacoustic response to sinusoidally modulated heating. A transfer matrix method for calculation of the thermoelastic response of multilayered samples exposed to modulated laser heating was presented by Bozoki et al. [14].

As a base for ultrasound pulse shaping by a pulsed laser, in present work we model, from fundamental principles and for one light pulse, the transient acoustical pulse generation and transmission in a light-absorbing layer. This layer is consisting of a stack of planar isotropic films, and is surrounded by two material layers that can have different physical properties. A Laplace transform approach is used in solving the set of linear, one-dimensional wave equations describing the transient wave propagation in the three-layer structure. The analytical solutions for pressures are compared to simulations of pressures as well as to experimental transducer voltage values. To facilitate the latter comparison, a system identification process of tranducer characteristics based on physical principles, is employed for estimations of the pressure to voltage transition.

\section{Analytical modelling}

We will analytically express laser-induced ultrasound as acoustic pressure $p=$ $p_{i}(x, t)$ in position $x$ at time $t$ in a material layer $i$. The analysis is based on a model presented by Shan et al. [15], of a light-absorbing layer within a fluid. 
Table 2.1: Denotations

\begin{tabular}{ll}
\hline Denotation & Description \\
\hline$\beta_{i}$ & isobaric volume expansion coefficient \\
$C_{p i}$ & isobaric heat capacity per unit mass \\
$\mu_{a i}$ & optical absorption coefficient \\
$E_{0 i}$ & energy density at layer leftmost surface \\
$\theta(t)$ & Heaviside step function \\
$\rho_{i}$ & density \\
$h_{i}$ & thickness of layer $\mathrm{i}, h_{i}=b_{i, i+1}-b_{i-1, i}$ \\
\hline
\end{tabular}

Present work is an extension that enables the properties of the materials on each of the two sides of the light-absorbing layer to be different. In the presented study we only consider one space dimension of the wave propagation problem. It is assumed, for the photoacoustic $1 \mathrm{D}$ wave equation that the material in each layer has isotropic properties and is linearly elastic. We assume that the laser pulse width $\tau_{\text {pulse }}$ is very short, so that the heat conduction is negligible during $\tau_{\text {pulse }}$ for thermal confinement, and also so that time $\tau_{\text {stress }}$ of pressure propagation at sound speed $c$ across a characteristic dimension $d_{c}$ of the heated region as $\tau_{\text {stress }}=d_{c} / c$ [16], fulfills the condition $\tau_{\text {pulse }} \ll \tau_{\text {stress }}$ for stress confinement. Further, acoustic attenuation is neglected in each layer.

To model the generation and propagation of laser-induced pressure waves, a photoacoustic wave equation is set up for each material layer $i$. For descriptions of coming denotations, see table 2.1. The index $i$ numbering of the layers is illustrated in Figure 2.1, where the index counting starts from the layer closest to the laser source. The boundaries around the light absorbing layer 2 are $x=b_{1,2}$ and $x=b_{2,3}$, where $0 \leq b_{1,2}<b_{2,3}$. The $1 \mathrm{D}$ photoacoustic wave equation [16] in layer $i=1,2,3$ for pressure $p_{i}(x, t)$ is

$$
\frac{\partial^{2} p_{i}(x, t)}{\partial x^{2}}-\frac{1}{c_{i}^{2}} \frac{\partial^{2} p_{i}(x, t)}{\partial t^{2}}=-\frac{\beta_{i}}{C_{p i}} \frac{\partial H_{i}(x, t)}{\partial t}
$$

were the right hand side source term holds the heating function

$$
H_{1} \equiv 0, H_{2}(x, t)=\mu_{a 2} \mathrm{e}^{-\mu_{a 2} x} E_{02} \delta(t), H_{3} \equiv 0,
$$

with light-absorption in layer $i=2$. In stress confinement, laser-pulse width is approximated by a delta function $\delta(t)$.

Initial conditions are

$$
\begin{aligned}
p_{i}\left(x, 0_{-}\right) & =0 \\
\frac{\partial}{\partial t} p_{i}\left(x, 0_{-}\right) & =0 \\
H_{2}\left(x, 0_{-}\right) & =0,
\end{aligned}
$$

and boundary conditions

$$
p_{i}\left(b_{i, i+1}, t\right)=p_{i+1}\left(b_{i, i+1}, t\right)
$$




$$
-\frac{1}{\rho_{i}} \frac{d}{d x} p_{i}\left(b_{i, i+1}, t\right)=-\frac{1}{\rho_{i+1}} \frac{d}{d x} p_{i+1}\left(b_{i, i+1}, t\right),
$$

are continuity in pressure and interface acceleration across each interface $x=$ $b_{i, i+1}$ between layer $i$ and $i+1$.

The set of $i$ photoacoustic wave equations 2.1, including boundary and initial conditions in equations $2.3-2.7$, are Laplace transformed

$$
L\left\{p_{i}(x, t)\right\}(t \rightarrow s)=y_{i}(x, s),
$$

and solved in the transform plane. General solutions with coefficients $C_{i F}(s)$ and $C_{i B}(s)$ for, in $x$-direction, forward and backward propagating waves in layer i respectively, are for layer $i=1,2,3$

$$
\begin{gathered}
y_{1}(x, s)=C_{1 B}(s) \mathrm{e}^{\frac{s x}{c_{1}}} \\
y_{2}(x, s)=C_{2 F}(s) \mathrm{e}^{-\frac{s x}{c_{2}}}+C_{2 B}(s) \mathrm{e}^{\frac{s x}{c_{2}}}+\frac{F_{2} s \mathrm{e}^{-\mu_{a 2} x}}{s^{2}-c_{2} \mu_{a 2}{ }^{2}} \\
y_{3}(x, s)=C_{3 F}(s) \mathrm{e}^{-\frac{s x}{c_{3}}},
\end{gathered}
$$

where we used that the indirect condition on bounded pressure in $x= \pm \infty$ gives $C_{1 F} \equiv 0$ and $C_{3 B} \equiv 0$. Material parameters are assembled into

$$
F_{2}=\frac{\mu_{a 2} \beta_{2} E_{02} c_{2}^{2}}{C_{p 2}}
$$

The coefficients $C_{i F}(s)$ and $C_{i B}(s)$ are obtained from an equation system of the two boundary conditions for each of the two boundaries. Of interest for comparison with measurements is pressure wave in layer 3. Corresponding coefficient $C_{3 F}(s)$ is

$$
C_{3 F}(s)=\mathrm{e}^{\frac{s b_{2,3}}{c_{3}}}\left(C_{2 F}(s) \mathrm{e}^{-\frac{s b_{2,3}}{c_{2}}}+C_{2 B}(s) \mathrm{e}^{\frac{s b_{2,3}}{c_{2}}}+\frac{s F_{2} \mathrm{e}^{-\mu_{a 2} b_{2,3}}}{s^{2}-c_{2}{ }^{2} \mu_{a 2}{ }^{2}}\right)
$$

with

$$
\begin{gathered}
C_{2 F D}(s)=\frac{C_{2 F}(s)}{D}, C_{2 B D}(s)=\frac{C_{2 B}(s)}{D} \\
D=\mathrm{e}^{\frac{s b_{1,2}}{c_{2}}}\left(\frac{\rho_{1} c_{1}}{\rho_{2} c_{2}}-1\right)-\mathrm{e}^{\frac{s\left(2 \cdot b_{2,3}-b_{1,2}\right)}{c_{2}}} \frac{\left(\frac{\rho_{1} c_{1}}{\rho_{2} c_{2}}+1\right)\left(\frac{\rho_{3} c_{3}}{\rho_{2} c_{2}}+1\right)}{\left(\frac{\rho_{3} c_{3}}{\rho_{2} c_{2}}-1\right)} \\
C_{2 F D}(s)=\left(\frac{\left(\frac{\rho_{1} c_{1}}{\rho_{2} c_{2}}-1\right)}{\left(\frac{\rho_{3} c_{3}}{\rho_{2} c_{2}}-1\right)} F_{2} \mathrm{e}^{-\mu_{a 2} b_{2,3}}\right)\left(\mathrm{e}^{\frac{s\left(b_{1,2}+b_{2,3}\right)}{c_{2}}} \frac{-\frac{\rho_{3} c_{3}}{\rho_{2} \mu_{2}} \mu_{a 2} c_{2}+s}{s^{2}-c_{2}{ }^{2} \mu_{a 2}{ }^{2}}\right)+ \\
+\left(\frac{\left(\frac{\rho_{3} c_{3}}{\rho_{2} c_{2}}+1\right)}{\left(\frac{\rho_{3} c_{3}}{\rho_{2} c_{2}}-1\right)} F_{2} \mathrm{e}^{-\mu_{a 2} b_{1,2}}\right)\left(\mathrm{e}^{\frac{s \cdot 2 b_{2,3}}{c_{2}}} \frac{s+\frac{\rho_{1} c_{1}}{\rho_{2} c_{2}} \mu_{a 2} c_{2}}{s^{2}-c_{2}{ }^{2} \mu_{a 2}{ }^{2}}\right)
\end{gathered}
$$




$$
\begin{array}{r}
C_{2 B D}(s)=\left(F_{2} \mathrm{e}^{-\mu_{a 2} b_{1,2}}\right)\left(\frac{s+\frac{\rho_{1} c_{1}}{\rho_{2} c_{2}} \mu_{a 2} c_{2}}{s^{2}-c_{2}{ }^{2} \mu_{a 2}{ }^{2}}\right)+ \\
+\left(\frac{\left(\frac{\rho_{1} c_{1}}{\rho_{2} c_{2}}+1\right)}{\left(\frac{\rho_{3} c_{3}}{\rho_{2} c_{2}}-1\right)} F_{2} \mathrm{e}^{-\mu_{a 2} b_{2,3}}\right)\left(\mathrm{e}^{\frac{s\left(b_{2,3}-b_{1,2}\right)}{c_{2}}} \frac{-\frac{\rho_{3} c_{3}}{\rho_{2} c_{2}} \mu_{a 2} c_{2}+s}{s^{2}-c_{2}{ }^{2} \mu_{a 2}{ }^{2}}\right)
\end{array}
$$

The denominator expression $D$, equation 2.15, is indicating periodic behaviour in the time-plane. The period do not start at $\mathrm{t}=0$ as for table inverse transform expression [17], so we chose to handle $D$ by means of a geometric series expansion $[17]$

$$
\frac{1}{D}=\sum_{j_{D}=1}^{\infty} \frac{(-1)}{\left(\frac{\rho_{1} c_{1}}{\rho_{2} c_{2}}-1\right)}\left(\frac{\left(\frac{\rho_{1} c_{1}}{\rho_{2} c_{2}}-1\right)\left(\frac{\rho_{3} c_{3}}{\rho_{2} c_{2}}-1\right)}{\left(\frac{\rho_{1} c_{1}}{\rho_{2} c_{2}}+1\right)\left(\frac{\rho_{3} c_{3}}{\rho_{2} c_{2}}+1\right)}\right)^{j_{D}} \mathrm{e} \mathrm{e}^{\left(\frac{s\left(j_{D} \cdot 2\left(b_{1,2}-b_{2,3}\right)-b_{1,2}\right)}{c_{2}}\right)} .
$$

Higher order expansion terms in $j_{D}$ are related to multiple reflections on layer interfaces, in an overlapping manner.

By means of equation 2.18, inverse transformation gives analytical acoustic pressure

$$
\begin{gathered}
p_{3}(x, t)=\sum_{j=1}^{N} p_{3_{j}}(x, t), \\
p_{3_{j}}(x, t)=\frac{F_{2}\left(R_{23}+1\right)}{2} \theta\left(x-b_{2,3}\right)\left(p_{3 j \text { even }}(x, t)+p_{3 j \text { odd }}(x, t)\right)
\end{gathered}
$$

where the Heaviside function $\theta\left(x-b_{2,3}\right)$ specifies the $x$ domain for layer $i=3$. Reflection coefficients of waves from layer $i=2$ reflected back into layer 2 , at interfaces towards layer 1 and 3 , are respectively

$$
R_{21}=\frac{c_{1} \rho_{1}-c_{2} \rho_{2}}{c_{2} \rho_{2}+c_{1} \rho_{1}}, R_{23}=\frac{c_{3} \rho_{3}-c_{2} \rho_{2}}{c_{2} \rho_{2}+c_{3} \rho_{3}} .
$$

Terms in equation 2.20 are

$$
\begin{array}{r}
p_{3 \text { jeven }}(x, t)=\left(R_{21} R_{23}\right)^{j-1} \mathrm{e}^{\mu_{a 2} c_{2}\left(t_{\text {jeven }}+t-\frac{b_{2,3}}{c_{2}}\right) .} \\
\theta\left(t_{\text {jeven }}+t\right) \theta\left(-t_{\text {jeven }}\right) \theta\left(-t_{\text {jeven }}-t-\frac{b_{1,2}-b_{2,3}}{c_{2}}\right), \\
p_{3 \text { jodd }}(x, t)=\frac{\left(R_{21} R_{23}\right)^{j}}{R_{23}} \mathrm{e}^{c_{2} \mu_{a 2}\left(-t_{j \text { odd }}-t-\frac{b_{1,2}}{c_{2}}\right) .} \\
\theta\left(t_{j \text { odd }}+t\right) \theta\left(-t_{j \text { odd }}\right) \theta\left(-t_{j \text { odd }}-t-\frac{b_{1,2}-b_{2,3}}{c_{2}}\right) .
\end{array}
$$

The subscripts even and odd refers to the even and odd arguments $j_{\text {arg }}$ in

$$
t_{\text {arg }}\left(j_{\text {arg }}\right)=\frac{b_{2,3}-x}{c_{3}}+\frac{j_{\text {arg }}\left(b_{1,2}-b_{2,3}\right)}{c_{2}},
$$


denoting number of reflections within layer 2

$$
\begin{gathered}
t_{j \text { even }}=t_{\text {arg }}(2(j-1)), \\
t_{j \text { odd }}=t_{\text {arg }}(2 j-1) .
\end{gathered}
$$

The three Heaviside functions in equations 2.22 and 2.23 originates in Laplace transform, in Laplace transform condition, and limits the function domain towards next wave component in the sum in equation 2.19 respectively.

\section{Experiments}

For comparison with the analytical pressure model, experiments were performed on laser-induced ultrasound in semitransparent light-absorbing planar film stack structures, in which absorption coefficient and layer thickness were varied.

For experimental setup, see figure 3.1. Pulsed laser light is sent from the left in the figure, into a basin with de-ionized and ultra-purified water. The laser light source was a system NL 202/SH from EKSPLA, Lithuania, as a diode pumped, Q-switched, frequency doubled, Nd:YAG laser, of wavelength $\lambda=532$ $\mathrm{nm}$, emitting $9 \mathrm{~ns}$ pulses of energy about $0.2 \mathrm{~mJ} /$ pulse. To avoid excessive heating of the absorbent, the laser beam was expanded from a diameter of $0.8 \mathrm{~mm}$ to approximately $8 \mathrm{~mm}$. In the basin we placed a sample consisting of a semi-transparent light-absorbing layer mounted on a $3.15 \mathrm{~mm}$ thick glass substrate. As light-absorbing layer we used planar, dyed, non-conductive, semitransparent, Polyethylene Terephthalate (PET) polymer films. To vary the absorbing layer thickness in the samples, films where stacked together in a stack structure of one or more films. The films had a thickness of $50 \mu \mathrm{m}$ and optical absorption coefficients $\mu_{a 2}=11 \cdot 10^{3}, 18 \cdot 10^{3}, 27 \cdot 10^{3}$ and $44 \cdot 10^{3} \mathrm{~m}^{-1}$ respectively, and were manufactured by Johnson Laminating and Coating Inc., CA, USA.

With an incident laser light pulse on the polymer film structure, laser pulse energy is transitioned to an ultrasonic pulse through a thermoelastic process. The ultrasonic pulse propagates through the film structure, continues into the water-filled basin and is subsequently recorded by an immersed ultrasonic transducer, a broad-band, PVDF based with a centre frequency of $25 \mathrm{MHz}$, model IA-FM 25.3 by GE Sensing and Inspection Technologies Inc., Germany. The signal from the ultrasonic transducer was amplified by a JSR Ultrasonics DPR500 Dual Pulser/Receiver, JSR Ultrasonics, NY, USA, and then digitised by means of an oscilloscope, a Yokogawa DL9140L, Yokogawa Corp., Japan, sampling at $12.5 \mathrm{GHz}$ with 12 effective bit resolution. During the experiments, water temperature in the basin was $23.1 \pm 0.4^{\circ} \mathrm{C}$.

For each experimental run, data from 10 individual laser pulses were recorded for subsequent signal processing. The recorded voltage time signal was processed in Matlab, Mathworks Inc., MA, USA. To reduce the influence from noise, the ultrasonic pulses of interest are windowed and by means of Grennberg et al. [18] aligned to reduce the timing jitter, and then averaged. 


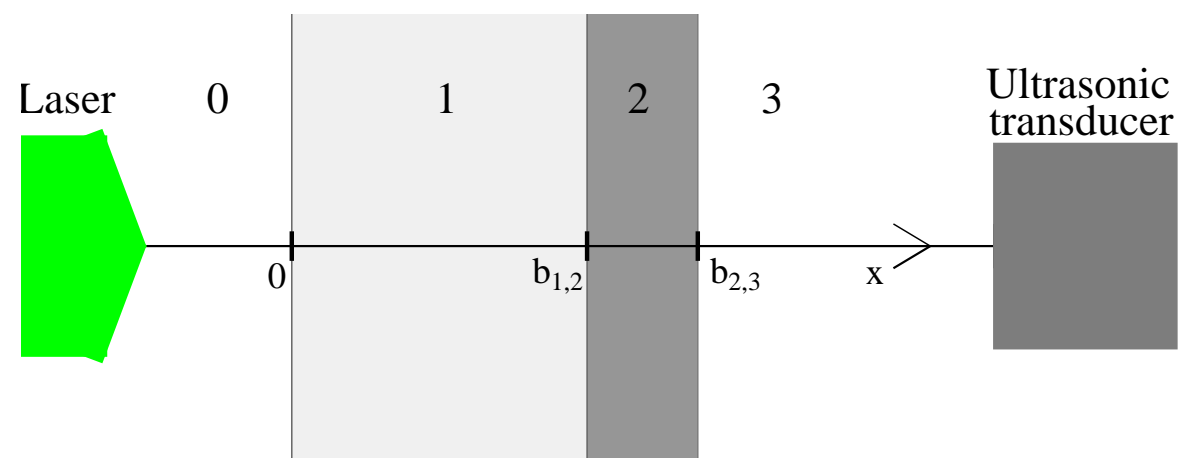

Figure 2.1: Illustration of material layers $i=0,1,2,3$. The light-absorbing layer2 has boundaries $x=b_{1,2}$ and $x=b_{2,3}$. Heating from a laser pulse induces ultrasound in layer 2 , which is recorded by an ultrasound transducer placed in material 3 .

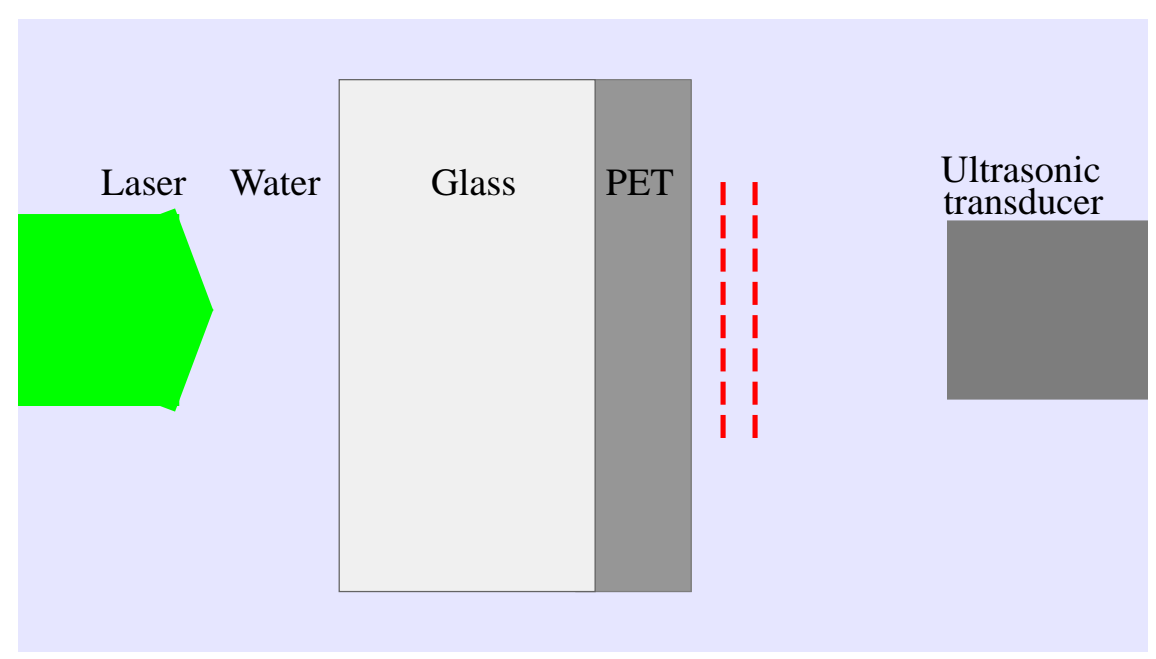

Figure 3.1: Experimental setup of pulsed laser light irradiating a semi-transparent lightabsorbing layer mounted on a glass substrate immersed in a basin of water. The laser-induced ultrasound (dashed lines) is recorded by an ultrasonic transducer for subsequent signal processing. 
Table 4.1: Physical parameter values

Sound speed of PET polymer film was calculated according to $c_{2}=\sqrt{Y / \rho_{2}}$ [20] with tensile elastic modulus $Y$ table value $(2.2-2.5) \cdot 10^{9} \mathrm{~Pa}[21]$. Absorption coefficient $\mu_{a 2}$ was calculated from manufacturer [22] specifications of film transmission $\mathrm{T}$ and reflection $\mathrm{R}$, as absorption $A=1-R-T$, together with Beer's law [23] of intensities $I(x)=I_{0} \mathrm{e}^{-\mu_{a 2} x}$, as the expression $\mu_{a 2}=-\ln (1-A) / h_{2}$ at film thickness $x=h_{2}$.

\begin{tabular}{|l|l|lll|}
\hline layer & parameter & material & value & reference \\
\hline 1 & & glass & & \\
& $c_{1}(\mathrm{~m} / \mathrm{s})$ & & $5.3 \cdot 10^{3}$ & {$[24]$} \\
& $\rho_{1}\left(\mathrm{~kg} / \mathrm{m}^{3}\right)$ & $2.5 \cdot 10^{3}$ & {$[24]$} \\
\hline 2 & & PET film & & \\
& $c_{2}(\mathrm{~m} / \mathrm{s})$ & & $1.3 \cdot 10^{3}$ & {$[20,21]$} \\
& $\rho_{2}\left(\mathrm{~kg} / \mathrm{m}^{3}\right)$ & $1.3 \cdot 10^{3}$ & {$[21]$} \\
& $E_{02}\left(\mathrm{~J} / \mathrm{m}^{2}\right)$ & 4 & \\
& laser energy $(\mathrm{J})$ & & $0.2 \cdot 10^{-3}$ & \\
& beam diameter $(\mathrm{m})$ & & $8 \cdot 10^{-3}$ & {$[21]$} \\
& $\mu_{a 2} \mathrm{~m}-1$ & & $(11,18,27,44) \cdot 10^{3}$ & \\
& $\beta_{2} \mathrm{~K}-1$ & & $8 \cdot 10^{-5}$ & {$[25]$} \\
& $C_{p 2}(\mathrm{~J} /(\mathrm{kg} \mathrm{K}))$ & & $1 \cdot 10^{3}$ & \\
& $h_{2}(\mathrm{~m})$ & & $(5,10,15,20) \cdot 10^{-5}$ & \\
\hline 3 & \multirow{2}{*}{3} & water at & $23.1 \pm 0.4{ }^{\circ} \mathrm{C}$ & \\
& $c_{3}(\mathrm{~m} / \mathrm{s})$ & & 1492 & {$[26]$} \\
& $\rho_{3}\left(\mathrm{~kg} / \mathrm{m}^{3}\right)$ & & 997.5 & {$[27,21]$} \\
\hline
\end{tabular}

\section{Simulations and Results}

The analytical model was evaluated for cases of physical parameters estimated for our experimental setup. The analytical pressures were compared to simulations from the MATLAB toolbox $k$-Wave, Treeby et al. [19]. The simulations were performed on initial pressure distributions similar to the photoacoustic source term in the inhomogeneous differential equation 2.1 for the analytical model. This initial pressure distribution was chosen from Gusev et al. [5]

$$
p_{2}(x, 0)=\mu_{a 2} E_{02} \Gamma \mathrm{e}^{-\mu_{a 2} x}
$$

with Grüneisen parameter

$$
\Gamma=c_{2}^{2} \beta_{2} / C_{p 2}
$$

In the analytical model, reflections only at interfaces with the middle number two layer are considered. However, in the simulations an additional layer $i=0$ is defined in emulation of the experimental setup of $i=0$ water, 1 glass, 2 PET, 3 water. Since the glass layer is acoustically thick in comparison to the PET film layer, acoustic reflections at boundary $b_{0,1}$ do not interfere with the ultrasound generated in the PET layer which is of our primary interest for comparisons.

Analytical and simulated photoacoustically generated pressures are illustrated in Figures 4.1 - 4.4. Figure 4.1 shows pressure from a layer of one light-absorbing film with the highest $\mu_{a 2}$, Figure 4.3 one film with the lowest $\mu_{a 2}$, and Figure 4.4 a stack of three films with the lowest $\mu_{a 2}$, and Figure 4.2 a stack of three films with the highest $\mu_{a 2}$. The oscillations in simulations from $k$-Wave after 
a discontinuity found in Figures 4.1 - 4.4 are artefacts due to representation of a very sharp edge by a finite number of complex exponentials. Figure 4.3 represents results from a thin layer of low absorption coefficient. Since less amount of the light is absorbed, the exponential behaviour gets less distinct.

\section{Model of the PVDF transducer}

We used the 'grey box' model presented by Shan et al. [28] in modelling our PVDF transducer. Experimental values of the transducer output voltage $v(t)$ were compared to the analytical solutions of the predicted pressure wave $p(t)$. The voltage output response to pressure input may be described by a secondorder differential equation

$$
\frac{d^{2} v(t)}{d t^{2}}+2 \zeta \omega_{n} \frac{d v(t)}{d t}+\omega_{n}^{2} v(t)=\epsilon \frac{d p(t)}{d t}
$$

where $\omega, \zeta$ and $\epsilon$ are constants. The constants $\zeta$ and $\omega$ are determined by the mechanical properties of the transducer film and backing material while constant $\epsilon$ is dependent on the piezoelectric and dielectric properties of the transducer. The constants are difficult to determine individually for a particular device as their interrelationships are complicated [28]. The parameters can instead be determined by a system identification method comparing experimental measurements and predicted pressure waves. A Z-transform is used on equation 5.1 to derive the recurrence relation between the voltage and pressure

$$
v(n)=K_{1} v(n-1)+K_{2} v(n-2)+K_{0}(p(n)-p(n-1))
$$

where $n$ is the current sample $\mathrm{n}=1, \ldots, \mathrm{N}$. The coefficients are functions of $\omega_{n}$, $\zeta, \epsilon$ and the sampling period $\tau$ such that

$$
\begin{aligned}
& K_{0}=\frac{\epsilon \tau}{1+2 \zeta \omega_{n} \tau+\omega_{n}^{2} \tau^{2}} \\
& K_{1}=\frac{2\left(1+\zeta \omega_{n} \tau\right)}{1+2 \zeta \omega_{n} \tau+\omega_{n}^{2} \tau^{2}} \\
& K_{2}=\frac{-1}{1+2 \zeta \omega_{n} \tau+\omega_{n}^{2} \tau^{2}}
\end{aligned}
$$

The least square method (LSM) was applied on equation 5.2 to solve the coefficients $K_{0}, K_{1}$ and $K_{2}$ for a collection of $\mathrm{N}$ samples during a sampling period. The relation of the system can be written in matrix form as

$$
V=\Phi b
$$

where the voltage $V$ is put into a $1 \times N$ vector and the other relations in equation 5.2 into a $3 \times N$ matrix denoted $\boldsymbol{\Phi}$ such that

$$
\begin{aligned}
\boldsymbol{V} & =[v(1), \ldots, v(N)]^{T} \\
\boldsymbol{\Phi} & =[\varphi(1), \ldots, \varphi(N)]^{T} \\
\varphi & =[v(n-1), v(n-2), p(n)-p(n-1)]
\end{aligned}
$$




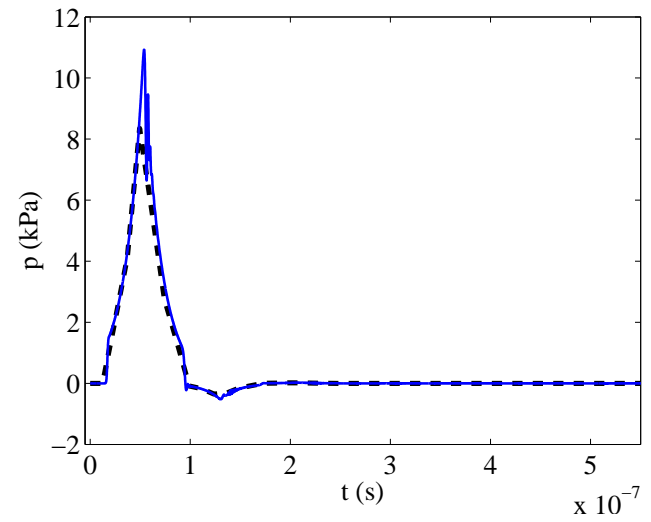

Figure 4.1: Pressure from analytical model (black dashed), and $k$-Wave simulated (blue line), for experiment material parameters of absorption coefficient and layer thickness $\mu_{a 2}=44 \cdot 10^{4}$ $\mathrm{m}^{-1}, h_{2}=5 \cdot 10^{-5} \mathrm{~m}$.

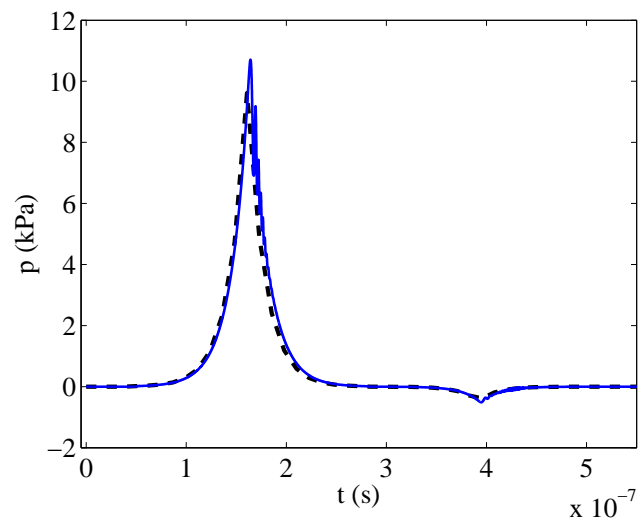

Figure 4.2: Pressure from analytical model (black dashed), and $k$-Wave simulated (blue line), for experiment material parameters of absorption coefficient and layer thickness $\mu_{a 2}=44 \cdot 10^{4}$ $\mathrm{m}^{-1}, h_{2}=15 \cdot 10^{-5} \mathrm{~m}$. 


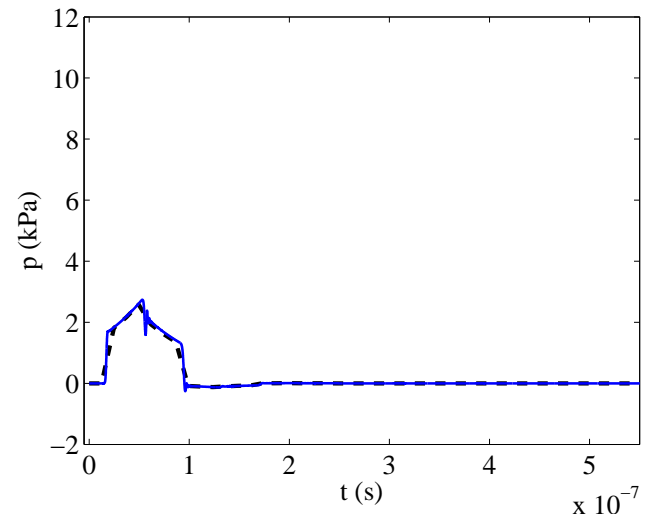

Figure 4.3: Pressure from analytical model (black dashed), and $k$-Wave simulated (blue line), for experiment material parameters of absorption coefficient and layer thickness $\mu_{a 2}=11 \cdot 10^{4}$ $\mathrm{m}^{-1}, h_{2}=5 \cdot 10^{-5} \mathrm{~m}$.

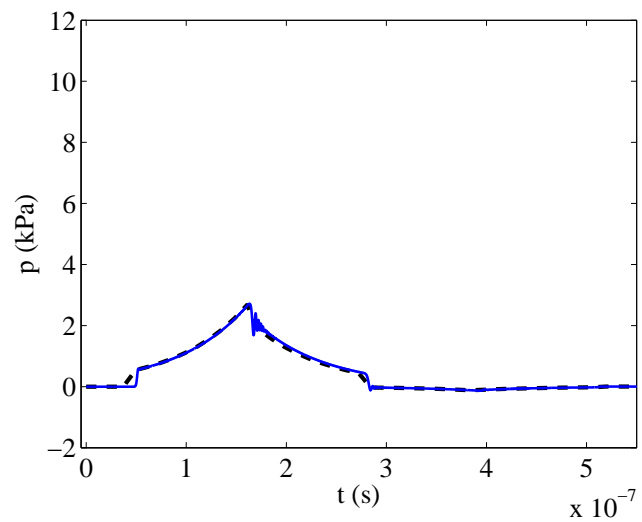

Figure 4.4: Pressure from analytical model (black dashed), and $k$-Wave simulated (blue line), for experiment material parameters of absorption coefficient and layer thickness $\mu_{a 2}=11 \cdot 10^{4}$ $\mathrm{m}^{-1}, h_{2}=15 \cdot 10^{-5} \mathrm{~m}$. 
For an over-determined system the least-square estimate is

$$
\boldsymbol{b}=\left(\boldsymbol{\Phi}^{T} \boldsymbol{\Phi}\right)^{-1} \boldsymbol{\Phi}^{T} \boldsymbol{V}
$$

where the elements in the vector $\boldsymbol{b}$ can be identified in equation 5.2

$$
\boldsymbol{b}=\left(\begin{array}{l}
K_{1} \\
K_{2} \\
K_{0}
\end{array}\right)
$$

about $2 \mathrm{~mm}$, in comparison to the thinner thicknesses of 0.05-0.02 $\mathrm{mm}$ used here, which might be a source of differences in the results. Datasets from eleven experimental runs with varied absorption coefficients and absorbing layer thicknesses were compared to corresponding analytical solutions. That is, in the system identification, experimental voltage values were compared to modelled pressures. In table 5.1, the result from the system characterisation is shown. The comparisons were unstable, though, such that when the experimental voltage values were moved just one sample in comparison to the analytical pressure values in the finding of the LS minimum error estimations of $\boldsymbol{b}$, especially $K_{0}$ fluctuated. For amplitude normalisation, the parameter $K_{0}$ was scaled. Individual ultrasonic transducer may differ from each other, and also the signal amplification may vary, which both affect $K_{0}$.

Figures 5.1 and 5.2 show comparisons between experimentally obtained and simulated voltages from analytical pressures in Figures 4.3 and 4.4 respectively, with a subsequent pressure to voltage estimation stage employing the procedure described above, but with the current experimental values not included in the respective coefficient estimation. In Figure 5.1 a single film is used as a light absorbing layer and the simulated and experimentally obtained voltages are seen to correspond quite well. In Figure 5.2 the number of films are increased to three to make up the light-absorbing layer. Figure 5.2 in comparison to Figure 5.1 shows that the correspondence between experimental and simulated voltages are lower for a stack of three films than for the situation with one light absorbing film.

\section{Discussion and Conclusion}

The 1D analytical model for heat-induced ultrasound in a light-absorbing layer seems to give pressures consistent with results from simulations. Higher pressure amplitude was indicated for a higher optical absorption coefficient, and longer pressure pulse length indicated for a larger layer structure thickness. The analytical modelling of the photoacoustic wave equation using the Laplace transform approach could easily be expanded into more layers. However this will result in an increasingly complex situation, unless using/finding a calculation tool of a more assistance. Parts of the Laplace transformed solution of the photoacoustic wave equation, identified as periodic by its denominator, were handled by means of geometric series expansion. In solving the photoacoustic wave equation for more than three layers, we suggest nested geometric series expansions for the corresponding Laplace transformed denominator, unless reflections are handled separately or another solution approach is used. 
Table 5.1: Coefficients from equation 5.2, from the system identification of the PVDF transducer

\begin{tabular}{|c|c|c|}
\hline Coefficient & Transducer estimation & Dimension \\
\hline$K_{0}$ & $-73 \pm 3$ & $10^{-7} \mathrm{~V} / \mathrm{Pa}$ \\
$K_{1}$ & $1.990 \pm 0.007$ & \\
$K_{2}$ & $-0.990 \pm 0.007$ & \\
\hline
\end{tabular}

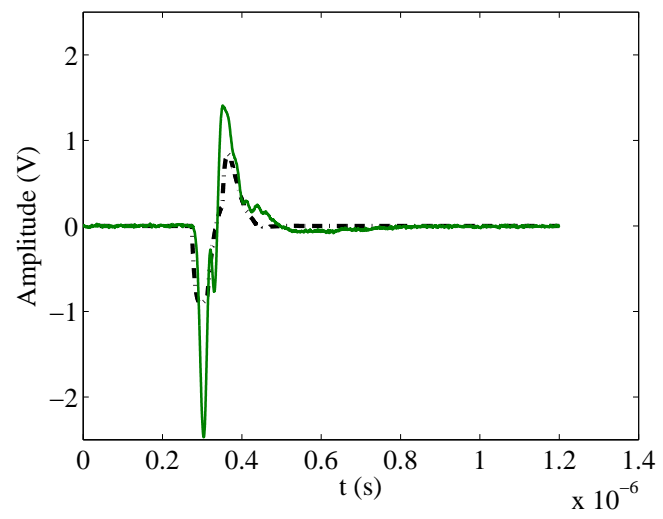

Figure 5.1: Experimental values on the voltage output from the transducer (green line), compared to LS-estimate (black dash-dot) of the voltage calculated from a simulated pressure profile using absorbing layer absorption coefficient $\mu_{a 2}=11 \cdot 10^{4} \mathrm{~m}^{-1}$ and layer thickness $h_{2}=5 \cdot 10^{-5} \mathrm{~m}$.

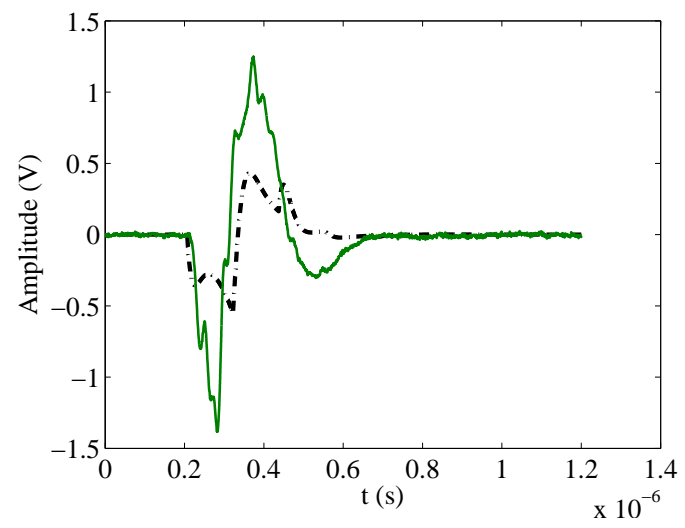

Figure 5.2: Experimental values on the voltage output from the transducer (green line), compared to LS-estimate (black dash-dot) of the voltage calculated from a simulated pressure profile using absorbing layer absorption coefficient $\mu_{a 2}=11 \cdot 10^{4} \mathrm{~m}^{-1}$ and layer thickness $h_{2}=15 \cdot 10^{-5} \mathrm{~m}$ 
The system identification used to model the PVDF transducer, for comparison between analytical pressure received by a certain individual of PVDF transducer and measured voltage values by that transceiver, appeared unstable for our set of measurements and simulations. In the photoacoustic wave equation, stress confinement $\tau_{\text {pulse }} \ll \tau_{\text {stress }}$ is assumed, but the pressure propagation time $\tau_{\text {stress }}$ through the heated region in present experiments is at its minimum value only about four times larger than the laser pulse-width $\tau_{\text {pulse }}$. This could possibly contribute to the difference between analytical model and the experiments via PVDF transducer system identification. Furthermore, the material parameters, see table 4.1, used in the model for our analytical pressures, are tabled values who may differ from the actual physical parameters in our experimental setup. The correspondence between experimental and simulated voltages seems lower for a stack of three films than for the situation with one light absorbing film. The presence of a thin adhesive and/or a coating layer could violate the assumption of material isotropy of the absorber and would affect the photoacoustic wave generation and propagation. Such additional layers could be included in the model by expanding the analytical pressure model into more layers. However, information on each layer's physical properties is needed to accurately model these additional layers.

Note that higher-dimension effects from e.g. waves in radial direction are not included in the analytical 1D model, but would be a part of the experimental voltage measurements.

\section{Acknowledgments}

This work was supported by grants from the Objective 2 Norra Norrland - EU Structural Funds, through ESiS and CMTF.

\section{References}

[1] L. V. Wang, S. Hu, Photoacoustic tomography: In vivo imaging from organelles to organs, Science 335 (2012) 1458-1462.

[2] S. Krishnaswamy, Ultrasonic characterization of the mechanical properties of thin films and coatings, in: T.-T. Wu, C.-C. Ma (Eds.), IUTAM Symposium on Recent Advances of Acoustic Waves in Solids, number 26 in IUTAM Bookseries, Springer Netherlands, 2010, pp. 77-86.

[3] S.-t. Luo, X.-l. Tan, M.-c. Pan, C.-g. Fan, Progress of laser-generated ultrasonic non-destructive testing technology (2011) 81924C-81924C.

[4] A. C. Tam, Applications of photoacoustic sensing techniques, Reviews of Modern Physics 58 (1986) 381-431.

[5] V. E. Gusev, A. A. Karabutov, Laser optoacoustics, AIP Press, New York, 1993.

[6] R. J. von Gutfeld, R. L. Melcher, 20MHz acoustic waves from pulsed thermoelastic expansions of constrained surfaces, Applied Physics Letters 30 (1977) 257-259. 
[7] T. Buma, M. Spisar, M. O’Donnell, High-frequency ultrasound array element using thermoelastic expansion in an elastomeric film, Applied Physics Letters 79 (2001) 548 .

[8] Y. Hou, S. Ashkenazi, S.-W. Huang, M. O'Donnell, Improvements in optical generation of high-frequency ultrasound, IEEE Transactions on Ultrasonics, Ferroelectrics and Frequency Control 54 (2007) 682-686.

[9] Y. Hou, J. S. Kim, S. Ashkenazi, M. O’Donnell, L. J. Guo, Optical generation of high frequency ultrasound using a two dimensional array of gold nanoparticles, in: IEEE Ultrasonics Symposium, 2006, IEEE, 2006, pp. 401-404. doi:10.1109/ULTSYM.2006.113.

[10] M. O’Donnell, Y. Hou, J.-S. Kim, S. Ashkenazi, S.-W. Huang, L. J. Guo, Optoacoustic generation of high frequency sound for 3-d ultrasonic imaging in medicine, The European Physical Journal Special Topics 153 (2008) 5358.

[11] H. Won Baac, J. G. Ok, H. J. Park, T. Ling, S.-L. Chen, A. J. Hart, L. J. Guo, Carbon nanotube composite optoacoustic transmitters for strong and high frequency ultrasound generation, Applied Physics Letters 97 (2010) 234104-234104-3.

[12] T. Sun, H. Chen, G. Diebold, Distributed source photoacoustic generation of ultrasound, Ultrasonics 32 (1994) 265-270.

[13] H. Hu, W. Zhang, J. Xu, Y. Dong, General analytical solution for photoacoustic effect with multilayers, Applied Physics Letters 92 (2008) 014103.

[14] Z. Bozoki, A. Miklos, D. Bicanic, Photothermoelastic transfer matrix, Applied Physics Letters 64 (1994) 1362.

[15] Q. Shan, A. Kuhn, P. Payne, R. Dewhurst, Characterisation of laserultrasound signals from an optical absorption layer within a transparent fluid, Ultrasonics 34 (1996) 629-639.

[16] L. V. Wang, H.-i. Wu, Biomedical optics : principles and imaging, WileyInterscience, Hoboken, N.J., 2007.

[17] D. Zwillinger, CRC standard mathematical tables and formulae., CRC Press, Boca Raton, 1996.

[18] A. Grennberg, M. Sandell, Estimation of subsample time delay differences in narrowband ultrasonic echoes using the hilbert transform correlation, IEEE Transactions on Ultrasonics, Ferroelectrics and Frequency Control 41 (1994) 588-595.

[19] B. E. Treeby, B. T. Cox, k-wave: MATLAB toolbox for the simulation and reconstruction of photoacoustic wave fields, Journal of Biomedical Optics 15 (2010) 021314-021314.

[20] L. E. Kinsler, A. R. Frey, A. B. Coppens, J. V. Sanders, Fundamentals of acoustics, Wiley, New York, 2000. 
[21] N. A. Waterman, M. F. Ashby, The Materials Selector, Chapman \& Hall, London, 1997.

[22] Johnson Window Films, Automotive window film renegade, http://www.johnsonwindowfilms.com/RN.php, (Retreived 2013-04-21). URL: http://www. johnsonwindowfilms . com/RN . php.

[23] M. Born, E. Wolf, Principles of optics : electromagnetic theory of propagation, interference and diffraction of light, Cambridge University Press, Cambridge, 1999.

[24] C. Nordling, J. Österman, Physics handbook for science and engineering, Studentlitteratur, Lund, 1999.

[25] J. Black, G. W. Hastings, F. Knovel, Handbook of biomaterial properties, 1st ed ed., Chapman \& Hall, London ; New York, 1998.

[26] J. Lubbers, R. Graaff, A simple and accurate formula for the sound velocity in water, Ultrasound in Medicine \& Biology 24 (1998) 1065-1068.

[27] D. R. Lide, CRC handbook of chemistry and physics: a ready-reference book of chemical and physical data, CRC Press, Boca Raton, Florida, 2004 .

[28] Q. Shan, A. Kuhn, R. Dewhurst, Characterization of polymer ultrasonic receivers by a photoacoustic method, Sensors and Actuators A: Physical 57 (1996) 187-195. 